\title{
Frequent Observations Accelerate Decay: The anti-Zeno Effect
}

\author{
A. G. Kofman and G. Kurizki \\ Department of Chemical Physics, Weizmann Institute of Science, 76100 Rehovot, Israel \\ Reprint requests to Prof. G. Ku.; E-mail: Gershon.Kurizki@weizman.ac.il \\ Z. Naturforsch. 56 a, 83-90 (2001); received February 6, 2001 \\ Presented at the 3rd Workshop on Mysteries, Puzzles and Paradoxes in Quantum Mechanics, \\ Gargnano, Italy, September 17 - 23, 2000.
}

\begin{abstract}
The quantum Zeno effect (QZE) is the striking prediction that the decay of any unstable quantum state can be inhibited by sufficiently frequent observations (measurements). The consensus opinion has upheld the QZE as a general feature of quantum mechanics which should lead to the inhibition of any decay. The claim of QZE generality hinges on the assumption that successive observations can in principle be made at time intervals too short for the system to change appreciably. However, this assumption and the generality of the QZE have scarcely been investigated thus far. We have addressed these issues by showing that (i) the QZE is principally unattainable in radiative or radioactive decay, because the required measurement rates would cause the system to disintegrate; (ii) decay acceleration by frequent measurements (the anti-Zeno effect - AZE) is much more ubiquitous than its inhibition. The AZE is shown to be observable as the enhancement of tunneling rates (e. g., for atoms trapped in ramped-up potentials or in current-swept Josephson junctions), fluorescence rates (e. g., for Rydberg atoms perturbed by noisy optical fields) and photon depolarization rates (in randomly modulated Pockels cells).
\end{abstract}

Key words: Quantum Decay; Quantum Measurements; Zeno-Effect; anti-Zeno-Effect; Quantum Tunneling.

\section{Introduction}

According to a prevailing view, claimed to be a general feature of quantum mechanics, successive frequent measurements must slow down the decay of any unstable system [1 - 11]. This is known as the Quantum Zeno Effect (QZE), introduced by Misra and Sudarshan [3], following the early work of Khalfin [1] and Fonda [2]. It has been colloquially phrased as follows: "a watched arrow never flies", or "a watched pot never boils" [8]. Recent estimates of the time interval between measurements required for the QZE inhibition of radiative decay have been $\tau_{\mathrm{Z}} \sim 3.6 \times 10^{-15} \mathrm{~s}[11]$. But is this view true?

We have recently shown [12] that, in fact, the opposite is mostly true for decay into open-space continua: The anti-Zeno effect (AZE), i.e. decay acceleration by frequent measurements, is far more ubiquitous than the QZE. This universal conclusion [13 - 15] has been described by our commentators in the words: "a watched pot boils faster" [16], "furtive glances trigger decay" [17]. Accordingly, our cartoonist shows that old Zeno, trying to reassure Gullielmo Tell and his boy that the arrow won't fly, is in for a nasty surprise (Figure 1). How can this conclusion be understood and what was missing in standard treatments that claimed the QZE universality?

\section{General Analysis}

We have reached the conclusion stated above by general analysis of measurement-affected decay. Consider $|e\rangle$, the measured state in a system ruled by the Hamiltonian $H=H_{0}+V$, where $V$ causes the coupling (decay) of $|e\rangle$ to all other eigenstates of $H_{0}$, to which we refer as the "reservoir" (Figure 2).

The probability amplitude $\alpha(t)$ to remain in $|e\rangle$, which has the energy $\hbar \omega_{a}$ obeys the following exact integro-differential equation

$$
\dot{\alpha}=-\int_{0}^{t} \mathrm{~d} t^{\prime} e^{i \omega_{a}\left(t-t^{\prime}\right)} \Phi\left(t-t^{\prime}\right) \alpha\left(t^{\prime}\right) .
$$

Here $\alpha(t)=\langle e \mid \Psi(t)\rangle e^{i \omega_{a} t}, \hbar \omega_{a}$ is the energy of $|e\rangle$, and 

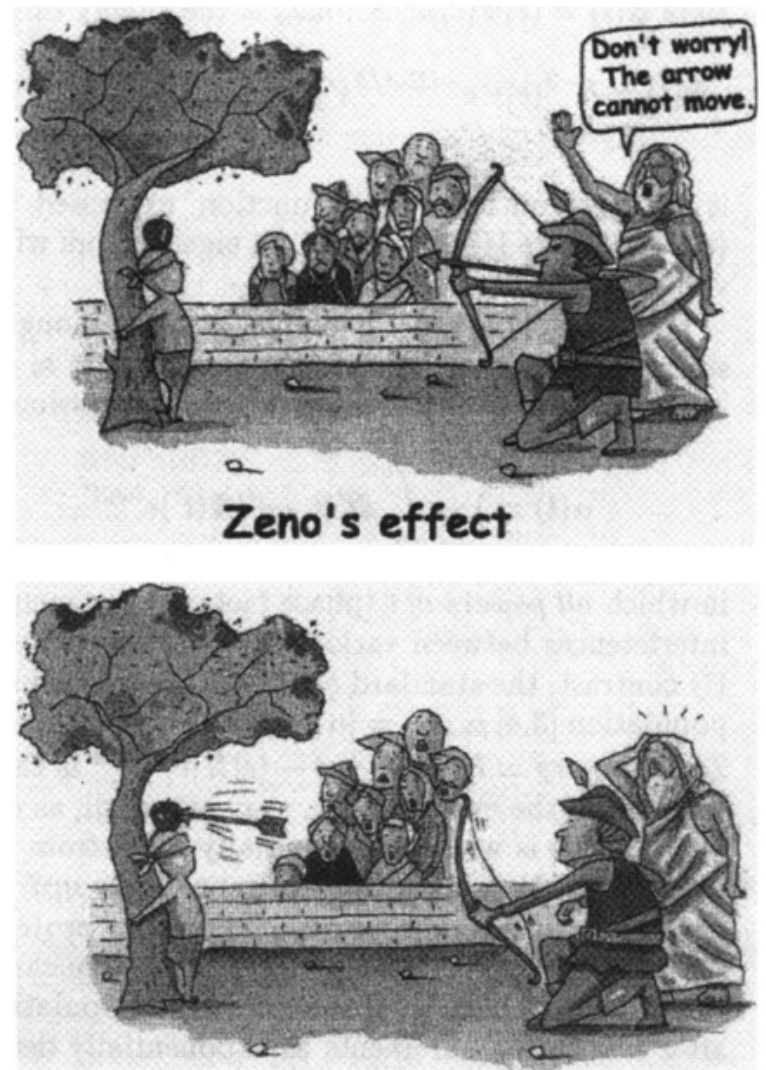

The anti-Zeno effect

Fig. 1. The Zeno and anti-Zeno effects as viewed by Zeno and William Tell watching an arrow in mid-air.

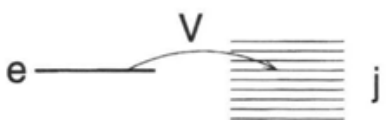

Fig. 2. The decay of a state into a "reservoir" via coupling.

$\Phi(t)=\hbar^{-2}\left\langle e\left|V e^{-i H_{0} t / \hbar} V\right| e\right\rangle=\hbar^{-2} \sum_{j}\left|V_{e j}\right|^{2} e^{-i \omega_{j} t}$

is the reservoir correlation function, expressed by $V_{e j}=\langle e|V| j\rangle$, where $|j\rangle(\neq|e\rangle)$ are $H_{0}$ eigenvectors with eigenvalues $\hbar \omega_{j}$.

Equation (1) is exactly soluble, but it is enough to consider its short-time behavior by setting $\alpha\left(t^{\prime}\right) \approx$ $\alpha(0)=1$ in the integral of (1). This yields the expression

$$
\alpha(t)=1-\int_{0}^{t} \mathrm{~d} t^{\prime}\left(t-t^{\prime}\right) \Phi\left(t^{\prime}\right) e^{i \omega_{a} t^{\prime}},
$$

in which all powers of $t$ (phase factors!) are included and interferences between various decay channels may occur. By contrast, the standard quadratic expansion in $t$ for the population $[3,4] \rho_{e e}(t)=$ $|\alpha(t)|^{2} \approx 1-t^{2} / \tau_{Z}^{2}$, in which the Zeno time $\tau_{\mathrm{Z}}=$ $\hbar /\left(\left\langle e\left|H^{2}\right| e\right\rangle-\langle e|H| e\rangle^{2}\right)^{1 / 2}$ is the inverse variance of the energy in $|e\rangle$, may often fail, as discussed below. This is where we essentially differ from standard treatments. How does this difference show up?

Consider instantaneous measurements - projections on $|e\rangle$ interrupting its decay at intervals $\tau$. We can use our result for $\alpha(t)$, (3) to express the population of $|e\rangle$ after $n$ such measurements as exponentially decaying at a rate $R, \rho_{e e}(t=n \tau)=|\alpha(\tau)|^{2 n} \approx$ $\exp (-R t)$. The universal form of $R$ is (in the frequency domain)

$$
R=2 \pi \int_{0}^{\infty} \mathrm{d} \omega G(\omega) F(\omega)
$$

This expression is the overlap of the reservoircoupling spectrum

$$
\begin{aligned}
G(\omega) & =\frac{1}{\pi} \operatorname{Re} \int_{0}^{\infty} \mathrm{d} t \Phi(t) e^{i \omega t} \\
& =\hbar^{-2} \sum_{j}\left|V_{e j}\right|^{2} \delta\left(\omega-\omega_{j}\right)
\end{aligned}
$$

and the measurement-induced broadening of the measured energy level

$$
F(\omega)=\frac{\tau}{2 \pi} \operatorname{sinc}^{2}\left(\frac{\left(\omega-\omega_{a}\right) \tau}{2}\right) .
$$

We can interpret the universal result (4) as an expression of the time-energy uncertainty relation for an unstable level with lifetime $\Delta t$, relating the energy broadening (uncertainty) of $|e\rangle$ to $\Delta t$, the interval between measurements (Fig. 3),

$$
\text { (4) } \Rightarrow \Delta E \Delta t \sim \hbar \text {. }
$$

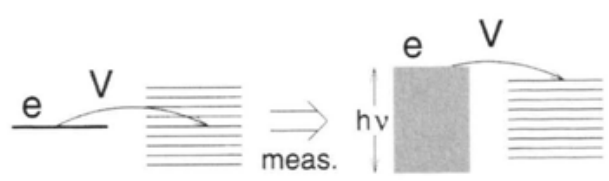

Fig. 3. Measurements broaden level $|e\rangle$, analogously to phase randomization by collisions at rate $\nu$, being drastically changing its decay into the reservoir. 


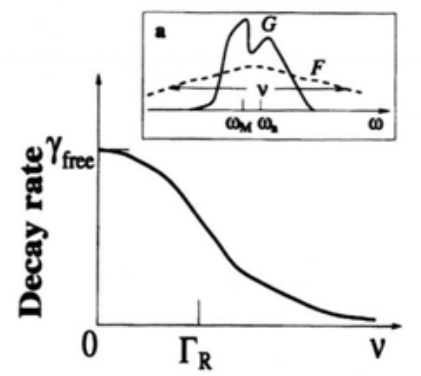

Fig. 4. Decay rate as a function of the measurement rate $\nu$, under the QZE conditions, shown in the inset, see (8), (15).

More generally, in (7) $\Delta t=1 / \nu$, where $\nu$ is a characteristic rate of measurements. With this definition, (7) holds both for ideal and nonideal (realistic) measurements. Relation (7) comes about since measurements (projections) dephase level $|e\rangle$, analogously to phase randomization by collisions, which induce a linewidth that is equal to the collision rate $\nu$.

A simple graphical analysis of the universal result (4) yields the main conclusions:

a) The QZE scaling (i.e., a decrease of the decay rate $R$ with an increase of $\nu$ ), is generally obtained when the measurement (dephasing) rate $\nu$ is much larger than the reservoir spectral width (Fig. 4):

$$
\nu \gg \Gamma_{R},\left|\omega_{\mathrm{a}}-\omega_{\mathrm{M}}\right| .
$$

Here $\Gamma_{R}$ is the reservoir width and $\omega_{\mathrm{M}}$ is the center of gravity of $G(\omega)$. In the special case of a peak-shaped $G(\omega)$, in (8) $\omega_{M}$ can be replaced by the position $\omega_{m}$ of the maximum. In the limit (8), one can approximate the spectrum $G(\omega)$ by a $\delta$-function, with a constant $C$ being the integrated spectrum,

$$
C=\int G(\omega) \mathrm{d} \omega=\left\langle V^{2}\right\rangle .
$$

This approximation becomes exact in the case of resonant Rabi oscillations $\left(\Gamma_{R}=0, \omega_{\mathrm{a}}=\omega_{\mathrm{M}}\right)$, which explains why the QZE is observable in Itano's experiment for any $\nu$. More generally, this approximation holds for any $G(\omega)$ that falls off faster than $1 / \omega$ on the wings. Then our universal expression yields the most general result for the QZE, namely that $R$ decreases with $\nu$ :

$$
R \approx 2 C / \nu
$$

where we defined generally

$$
\nu=\left[\pi F\left(\omega_{\mathrm{a}}\right)\right]^{-1}
$$

In particular, as follows from (6), $\nu=2 / \tau$ for instantaneous projections. The flattening of the spectral peak of $G(\omega)$ by the broad function $F(\omega)$ in the convolution is seen to be the origin of the QZE. To put it simply, if the system is probed frequently enough, the QZE arises since the effective decay rate is averaged over all decay channels, many of which are weak, due to the energy uncertainty incurred by the measurements.

This result contradicts the claim of QZE universality and demonstrates the failure of the standard quadratic expansion: Equation (8) shows that the QZE conditions can be much more stringent than the requirement to have $t \sim 1 / \nu \ll \tau_{\mathrm{Z}}$. The crucial point emphasized below is that (8) may be principally impossible to satisfy.

The above discussion presumes that the integral in (9) converges. However, when $G(\omega)$ falls off as $1 /\left|\omega-\omega_{\mathrm{M}}\right|$ or slower and hence $C$ is infinite, the QZE still holds under condition (8), except that $R$ decreases with $\nu$ more slowly than $\nu^{-1}$. This situation is illustrated by a reservoir with a slowly decreasing tail,

$$
G(\omega)=A\left|\omega-\omega_{\mathrm{c}}\right|^{-\beta} \text { for } s\left(\omega-\omega_{\mathrm{c}}\right) \gg \Gamma_{R}
$$

and $G(\omega)$ diminishes fast or is cut off for $s\left(\omega-\omega_{\mathrm{c}}\right)<$ $\Gamma_{R}$. Here $\omega_{\mathrm{c}}$ is the cutoff frequency, $0<\beta<1$, $\theta()$ is the unit step function, and $s$ can equal 1 or -1 . For instance, (12) approximately holds near the waveguide cutoff ( $s=1, \beta=1 / 2)$ or the vibrational Debye cutoff ( $s=-1, \beta=1 / 2)$. The QZE scaling for a reservoir response (12) is found to be

$$
R=B \nu^{-\beta} \text {. }
$$

Here $B=\left[2^{\beta} \pi / \cos (\pi \beta / 2) \Gamma(2+\beta)\right] A$, where $\Gamma()$ is the gamma-function [18] (in particular, $B=$ $\left(8 \pi^{1 / 2} / 3\right) A$ for $\left.\beta=1 / 2\right)$. Equation (13) holds for a sufficiently weak coupling $\left(A \ll \nu^{\beta+1}\right)$ and under condition (8), which, in the case of (12), reduces to $\left|\omega_{\mathrm{a}}-\omega_{\mathrm{c}}\right| \ll \nu$.

More generally, under condition (8), the QZE scaling of $R$ occurs for any $G(\omega)$ such that

$$
G(\omega) \rightarrow 0 \text { at } \omega \rightarrow \infty .
$$

Conditions (8) and (14) ensure the QZE scaling only for sufficiently large measurement rates and do not always imply a monotonous decrease of $R$ as $\nu$ increases (see Figure 4). The latter behavior, which is what one usually has in mind in discussions of 


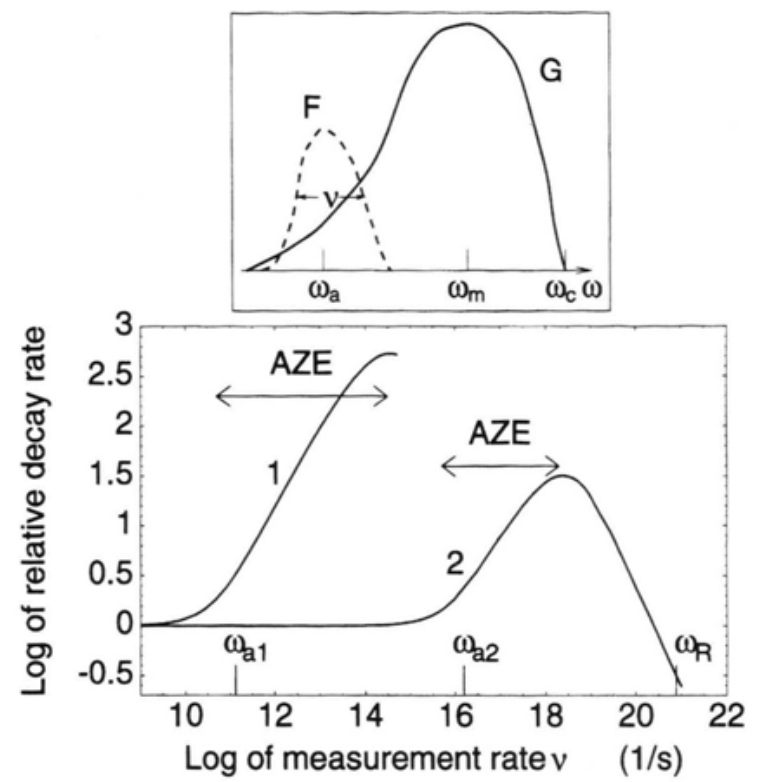

Fig. 5. AZE dependence of the decay rate. Inset: Condition for the AZE, (16). Graph: the dependence of the logarith of the normalized decay rate $\log _{10}\left(R / R_{\mathrm{RG}}\right)$ on $\log _{10} \nu$ for a spontaneously emitting hydrogenic state. The values of the atomic transition frequencies corresponding to curves 1,2 : $\omega_{\mathrm{a} 1}=1.32 \times 10^{11} \mathrm{~s}^{-1}, \omega_{\mathrm{a} 2}=1.55 \times 10^{16} \mathrm{~s}^{-1}$, whereas the relativistic cutoff $\omega_{\mathrm{R}}=7.76 \times 10^{20} \mathrm{~s}^{-1}$. The Bohr frequencies are $\omega_{\mathrm{B} 1}=1.22 \times 10^{15} \mathrm{~s}^{-1}, \omega_{\mathrm{B} 2}=8.50 \times 10^{18} \mathrm{~s}^{-1}$. The $\mathrm{AZE}$ ranges are marked.

the QZE, is obtained only in special situations. For instance, it occurs when $G(\omega)$ is a peak and $\omega_{\mathrm{a}}$ is within its width $\Gamma_{R}$, i. e.,

$$
\left|\omega_{\mathrm{a}}-\omega_{\mathrm{M}}\right| \lesssim \Gamma_{R}
$$

b) The opposite to the QZE scaling is obtained whenever $\omega_{\mathrm{a}}$ is significantly detuned from the nearest maximum of $G(\omega)$ at $\omega_{\mathrm{m}}$, so that $G\left(\omega_{\mathrm{a}}\right) \ll G\left(\omega_{\mathrm{m}}\right)$. Let $\nu$ be much less than the detuning of $\omega_{\mathrm{a}}$ from $\omega_{\mathrm{m}}$, though not too small (Fig. 5 - inset):

$$
\delta_{\mathrm{a}} \lesssim \nu \ll\left|\omega_{\mathrm{m}}-\omega_{\mathrm{a}}\right|,
$$

where $\delta_{\mathrm{a}}$ is the interval around $\omega_{\mathrm{a}}$ over which $G(\omega)$ changes appreciably. In the limit (16), the rate $R$ grows with $\nu$, since the dephasing function $F(\omega)$ is then probing more of the rising part of $G(\omega)$ in the convolution. This limit implies the anti-Zeno effect (AZE) of decay acceleration by frequent measurements. Physically, this means that, as the energy uncertainty increases with the measurement rate $\nu$, the state decays into more and more channels, whose weight $G(\omega)$ is progressively larger.

Remarkably, we may impose condition (16) in any reservoir that is not spectrally flat, by measurements at a rate comparable to the $\omega$ range over which $G(\omega)$ changes appreciably. This reveals the universality of the AZE, which we noted already for radiative decay in cavities [14].

c) In the limit $\nu \ll \delta_{\mathrm{a}}$, Eq. (4) yields the Golden Rule result (i.e., the normal decay rate in the absence of measurements)

$$
R_{\mathrm{GR}}=2 \pi G\left(\omega_{\mathrm{a}}\right)
$$

d) More subtle behavior occurs in the domain between the QZE and AZE limits. Assume, for simplicity, that $G(\omega)$ is single-peaked and satisfies condition (14). When $\nu$ increases from the limit (16) up to the range where the right-hand-side inequality is violated, then $\nu \gg\left|\omega_{\mathrm{m}}-\omega_{\mathrm{a}}\right|$, which is now equivalent to condition (8), implying the Zeno scaling of (10) or (13). But even in this QZE-scaling regime, $R$ remains larger than the Golden Rule rate $R_{\mathrm{GR}}(17)$, up to much higher $\nu$, as expressed by the following condition for "genuine $Q Z E$ "

$$
R<R_{\mathrm{GR}} \text { for } \nu>\nu_{\mathrm{QZE}}
$$

where

$$
\nu_{\mathrm{QZE}}=2 C / R_{\mathrm{GR}}=C / \pi G\left(\omega_{\mathrm{a}}\right)
$$

in the case of a finite $C$, and

$$
\nu_{\mathrm{QZE}}=\left(B / R_{\mathrm{GR}}\right)^{1 / \beta}=\left[B / 2 \pi G\left(\omega_{\mathrm{a}}\right)\right]^{1 / \beta}
$$

in the case of (12). The quantity $\nu_{\mathrm{QZE}}$ may turn out to be much greater than the boundary $\nu_{1}$ of the QZEscaling regime (see below).

The value of $\nu_{\mathrm{QZE}}$ given by (19a) was identified with the reciprocal "jump time" $1 / \tau_{J}$, i. e., the maximal time interval between measurements for which the decay rate is appreciably changed [19]. However, the correct value of the reciprocal jump time is $\delta_{a}$, which may be smaller by many orders of magnitude than $\nu_{\text {QZE }}$. In the special case of ideal instantaneous measurements and a Lorentzian $G(\omega)$, the genuineQZE condition (18) reduces to that of [20]. 
These considerations apply (with some limitations) to hydrogenic radiative decay (spontaneous emission), for which $G(\omega)$ can be calculated exactly [21]:

$$
G(\omega)=\frac{\alpha \omega}{\left[1+\left(\omega / \omega_{B}\right)^{2}\right]^{4}} .
$$

Here $\omega_{\mathrm{B}} \sim c / a_{\mathrm{B}}$, where $c$ is the vacuum light speed and $a_{\mathrm{B}}$ is the radius of the electron orbit. Then (4) and (6) may yield the AZE trend

$$
R \approx \alpha \nu\left[\ln \left(\omega_{\mathrm{B}} / \nu\right)+C_{1}\right]\left(\omega_{\mathrm{a}} \ll \nu \ll \omega_{\mathrm{B}}\right),
$$

where $C_{1}=0.354$ and $\nu=2 / \tau$. The AZE trend should be observable (Fig. 5) for $\nu \gtrsim \omega_{\mathrm{a}}$, i. e., for microwave Rydberg transitions on a ps scale (provided we can isolate one transition) and for optical transitions on the sub-fs scale. The boundary between the AZE and QZE-scaling regions is now given by $\nu_{1} \sim \omega_{\mathrm{B}}$ and the genuine-QZE condition (18) by $\nu>\nu_{\mathrm{QZE}} \sim \omega_{\mathrm{B}}^{2} / 12 \pi \omega_{\mathrm{a}} \gg \nu_{1}$ [cf. (19a)], rendering $R<R_{\mathrm{GR}}=2 \pi \alpha \omega_{\mathrm{a}}$. This analysis implies that the "genuine QZE" range $\nu>\nu_{\mathrm{QZE}}$ is principally unattainable, since it requires measurement rates above relativistic cutoff, which are detrimental to the system, leading to the production of new particles. A similar principal obstacle occurs for radioactive decay. By contrast, the AZE is accessible in decay processes, such as spontaneous emission or the nuclear $\beta$-decay, and can essentially always be imposed.

\section{Realistic Measurements}

Thus far we have assumed ideal instantaneous projections on $|e\rangle$. Does a more realistic description of measurements still support these results? The answer is positive for the two possible types of measurements of $|e\rangle$ :

a) Impulsive measurements. Such measurements are realizable, e.g., in Cook's scheme [6] implemented by Itano et al. [7] (Fig. 6): the decay process is repeatedly interrupted by a short pulse transferring the population of $|e\rangle$ to a higher auxiliary state $|u\rangle$, which then decays back fast enough to $|e\rangle$ incoherently. This case conforms to our universal result (4) to a very good approximation, with the dephasing function given by (6).

b) Continuous measurements should not be confused with the limit of vanishing intervals between successive continuous projections discussed by Misra

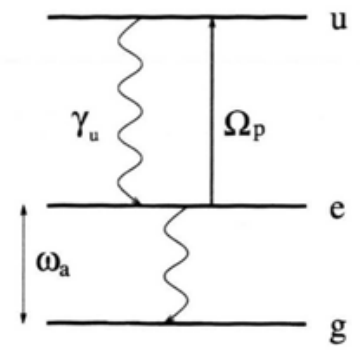

Fig. 6. The Cook scheme: see text for explanations.

and Sudarshan. This limit is unphysical, corresponding to an infinite energy spread $\hbar \nu$. In contrast, realistic continuous measurements, though monitoring the state incessantly, still require a finite time for completing an observation, i. e., they have a finite effective rate $\nu$.

This is seen for stationary dephasing when the $|e\rangle \leftrightarrow|u\rangle$ transition is driven by a near-resonant continuous-wave field, such that $1 / \Omega_{p} \gg 1 / \gamma_{u}$, yielding

$$
\nu=2 \Omega^{2} / \gamma_{u} .
$$

One can show that the universal result still holds for such measurements, with $F(\omega)$ being a Lorentzian centered at $\omega_{\mathrm{a}}$ :

$$
F(\omega)=\frac{1}{\pi} \frac{\nu}{\left(\omega-\omega_{\mathrm{a}}\right)^{2}+\nu^{2}} .
$$

Thus, qualitatively, there is no essential distinction between different frequent measurements: ideal projections, impulsive measurements, and continuous measurements.

\section{QZE and AZE for Photon Polarization Dephasing}

As an example, consider the decay of photon polarization into a "reservoir" created by the phase-randomizing element (polarization rotator) controlled by a noisy field. Measurements are effected by a polarization beam-splitter (PBS) and an absorber with transparency $\theta$ (see Fig. 7) [22].

Let us first assume constant phase jumps $\Delta \varphi$ at the polarization rotator. Uninterrupted evolution then corresponds to Rabi oscillations of the horizontal polarization probability (for $\theta=1$ ):

$$
P_{\mathrm{h}}(n)=\cos ^{2}(n \Delta \varphi) .
$$




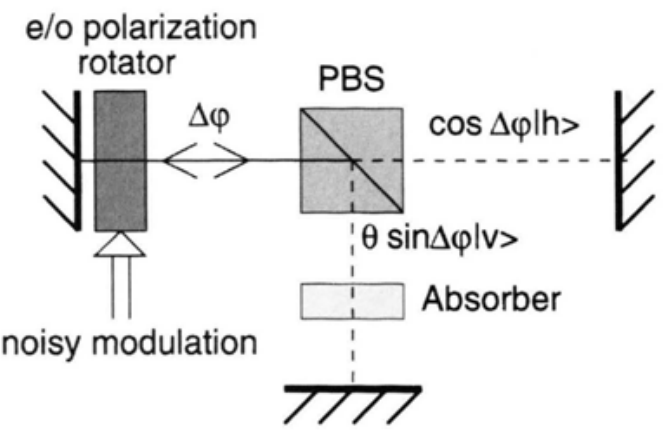

Fig. 7. Setup for controlling the polarization decay of a photon bouncing between the mirrors. Measurements are effected by a polarization beam-splitter (PBS) and an absorber with variable transparency $\theta$. The "reservoir" into which the polarization decays is realized upon modulating a Pockels cell (which rotates the polarization by $\Delta \varphi$ ) by a field with controllable noise prperties.

Decay via perfect (projective) measurements (for $\theta=0$ ) results in

$$
P_{h}(n)=e^{-n(\Delta \varphi)^{2}} .
$$

This decay is slower than Rabi oscillations, signifying the QZE. Imperfect (weak) measurements $(0<\theta<1)$ yield for small phase jumps $\left[(\Delta \varphi)^{2} \ll(1-\theta)^{2}\right]$ an exponential decay

$$
P_{h}\left(t=n \tau_{\mathrm{r}}\right)=\exp \left[-\frac{2(\Delta \varphi)^{2}}{\tau_{\mathrm{r}}^{2} \nu} t\right]
$$

$\tau_{\mathrm{r}}$ being the round-trip time. This decay still conforms with the QZE: the decay rate decreases with the measurement rate

$$
\nu=\frac{2(1-\theta)}{1+\theta} \frac{1}{\tau_{\mathrm{r}}} .
$$

We now proceed to discuss random phase jumps $\Delta \varphi_{n}$, caused by noisy modulation. We then obtain for sufficiently small jumps

$$
R=2 \pi \int_{-\pi / \tau_{\mathrm{r}}}^{\pi / \tau_{\mathrm{r}}} \mathrm{d} \omega G(\omega) F(\omega),
$$

which is an extension of (4) to such a "reservoir". Here $F(\omega)$ is peaked at $\omega=0$ and has the characteristic width (27).

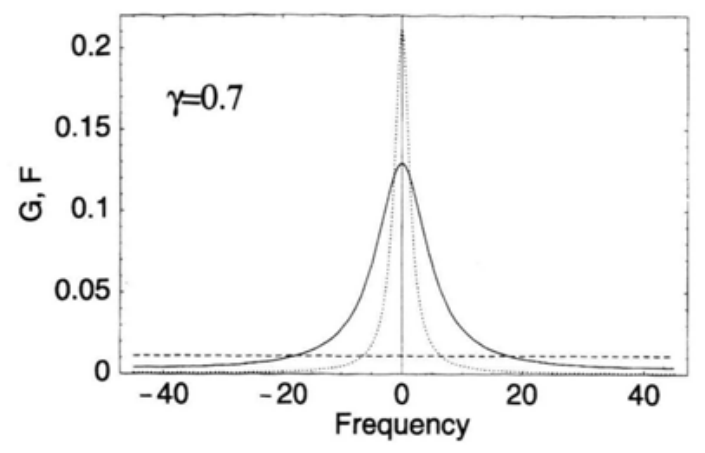

Fig. 8. The overlap of $G$ (solid line) and $F$ for correlated phase jumps in the setup of Fig. 7: see (18 - 34). Dashed line: $F(\omega)$ with $\theta=0$ (perfect projections). Dotted line: $F(\omega)$ with $\theta=0.9$ (ineffective measurements). Here $B=0.1$, $\tau_{\mathrm{r}}=0.07$.

The key parameter for $G(\omega)$ in (28) is the correlation of consecutive phase jumps:

$$
\left\langle\Delta \varphi_{n+1} \Delta \varphi_{n}\right\rangle=\gamma\left\langle\Delta \varphi_{n}^{2}\right\rangle .
$$

For highly correlated jumps $(\gamma \approx 1)$ we find

$$
G(\omega) \approx \frac{B^{2}}{\pi \tau_{\mathrm{r}}^{2}} \frac{\Gamma_{\mathrm{R}}}{\Gamma_{\mathrm{R}}^{2}+\omega^{2}},
$$

i. e. a Lorentzian of width

$$
\Gamma_{\mathrm{R}}=(1-\gamma) / \tau_{\mathrm{r}} .
$$

For highly anticorrelated jumps $(\gamma \approx-1)$ the "reservoir" spectrum is a sum of two shifted Lorentzians,

$$
G(\omega) \approx \sum_{j= \pm 1} \frac{B^{2}}{\pi \tau_{\mathrm{r}}^{2}} \frac{\Gamma_{\mathrm{R}}^{\prime}}{\Gamma_{\mathrm{R}}^{\prime 2}+\left(\omega+j \pi / \tau_{\mathrm{r}}\right)^{2}}
$$

of width

$$
\Gamma_{\mathrm{R}}^{\prime}=(1+\gamma) / \tau_{\mathrm{r}}
$$

If $G(\omega)$ is peaked at $\omega=0$ (the high-correlation case $\gamma \approx 1)$, then perfect measurements $(\theta=0)$, corresponding to a flat $F(\omega)$, will reduce $R$, causing the QZE trend, as compared to weak measurements $(\theta \approx 1)$, corresponding to a narrow spectral profile

$$
F(\omega) \approx \frac{1}{\pi} \frac{\nu}{\nu^{2}+\omega^{2}},
$$

with $\nu=(1-\theta) / \tau_{\mathrm{r}}$ (see Figure 8). 


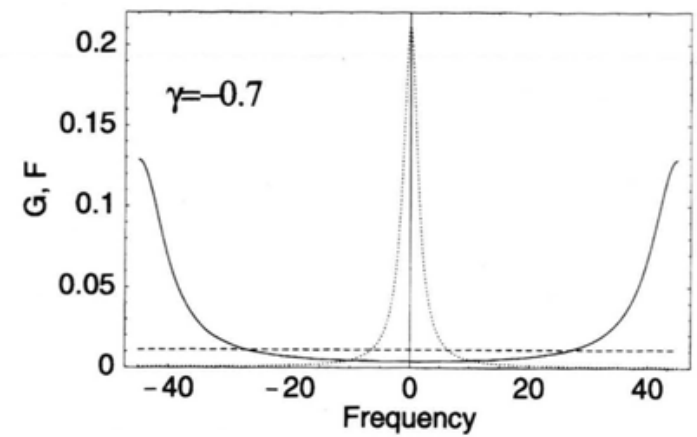

Fig. 9. Idem, for anticorrelated phase jumps.

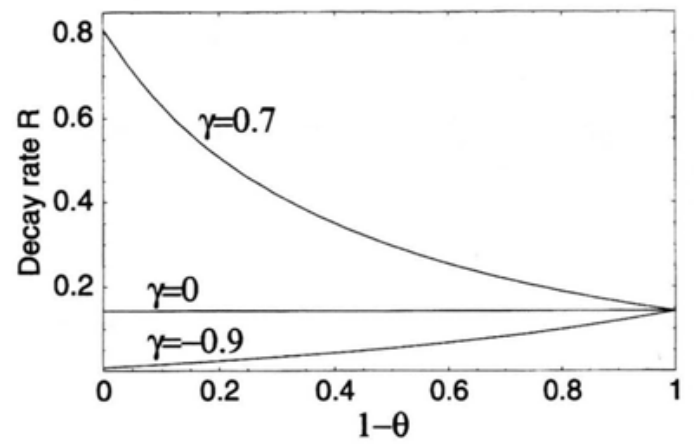

Fig. 10. The decay rate in the setup of Fig. 7 as a function of the measurement effectiveness $1-\theta$ (which is on the order of the dimensionless measurement rate $\nu \tau_{\mathrm{r}}$ ) for correlated $(\gamma=0.7)$, Markovian $(\gamma=0)$ and anticorrelated $(\gamma=-0.9)$ phase fluctuations.

The opposite (AZE) trend holds if $G(\omega)$ is peaked at $\omega= \pm \pi / \tau_{\mathrm{r}}$ (the highly anti-correlated case, $\gamma \approx-1$ ) (Figure 9). The two trends are illustrated in Figure 10.

\section{Atom Escape (Decay) by Tunneling from Accelerated Potential}

Theoretical and experimental studies by Raizen et al. [23] have shown that cold atoms trapped in an accelerated ("washboard"-shaped) periodic potential tunnel out (Fig. 11) non-exponentially, at times $t<\tau_{0} \equiv \epsilon_{g} /\left(\hbar k_{L} a\right)$, where $\epsilon_{g} \equiv \hbar \omega g$ is the band gap corresponding to the potential, $a$ the acceleration and

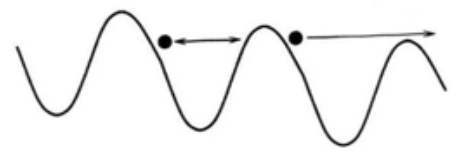

Fig. 11. Atoms tunnel out of a "washboard" potential.

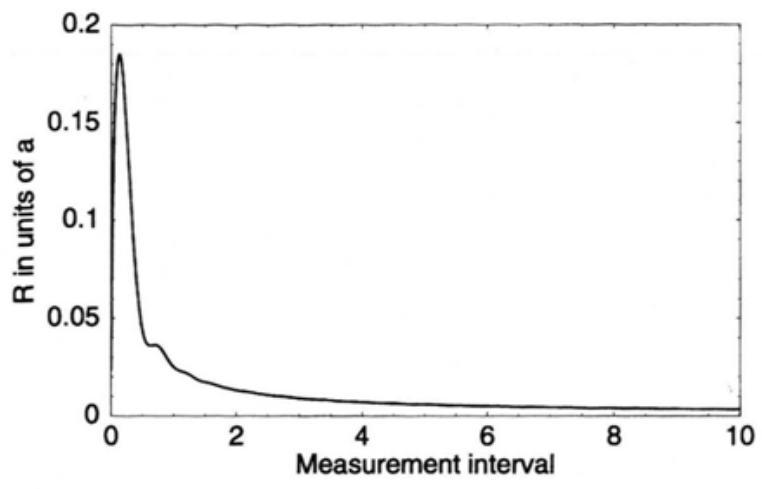

Fig. 12. The AZE trend for the decay rate $R$ (in units of $\tau_{0}^{-1}$ ) in a "washboard" potential (Fig. 11) as a function of the scaled measurement interval $\tau \tau_{0}\left(\omega_{\mathrm{g}}=10 / \tau_{0}\right.$, $M^{2} a / 8 \hbar^{2} k_{\mathrm{L}}^{3}=0.01$, where $M$ is the mass of the atom.

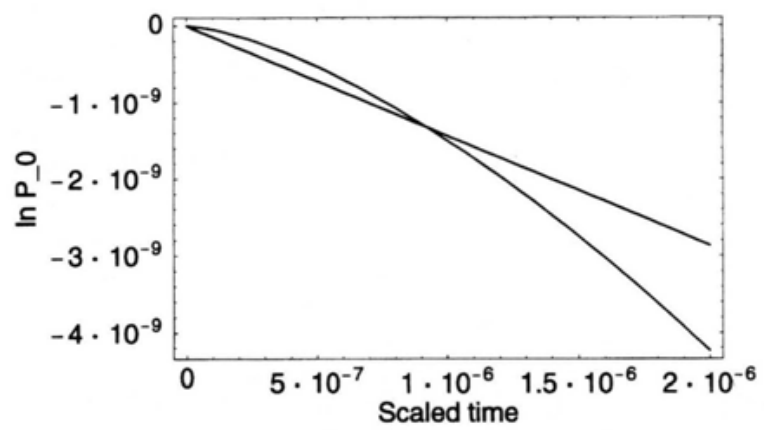

Fig. 13. The decay law in a "washboard" potential as a function of the scaled time $t / \tau_{0}\left(\omega_{\mathrm{g}}=10 / \tau_{0}, M^{2} a / 8 \hbar^{2} k_{\mathrm{L}}^{3}=\right.$ $0.01)$. Straight line: the exponential decay with the rate $R_{\mathrm{GR}}=3.2 \times 10^{-4} \tau_{0}^{-1}$.

$k_{L}$ the wave number of the laser creating this potential. Our prediction is that AZE should arise for measurement intervals $\tau \gg \hbar / \epsilon_{g}$ and QZE for $\tau \ll \hbar / \epsilon_{g}$. These trends are plotted in Figure 12. The two curves in Fig. 13 intersect at the time $2 / \nu_{\text {QZE }}$. Hence, in this case $2 / \nu_{\text {QZE }}\left(\sim 10^{-6} \tau_{0}\right)$ is much less than the boundary of the QZE-scaling region $\tau_{1} \sim 1 / \omega_{g}\left(=0.1 \tau_{0}\right)$ (see Figure 12).

\section{Experimental Accessibility}

Table 1 lists a number of processes where the AZE may be observed at accessible measurement intervals $t \sim \tau_{\mathrm{AZE}}$. By contrast, the QZE $\left(\tau_{\mathrm{QZE}} \ll \tau_{\mathrm{AZE}}\right)$ either is principally unobservable or, as a rule, is practically much less accessible than the AZE ( $\left.\tau_{\text {QZE }} \ll \tau_{\text {AZE }}\right)$. Notable exceptions, where the AZE and QZE are equally accessible, are the cases of a narrow (near- 
Table 1. Experimentally accessible processes with an AZE.

\begin{tabular}{|c|c|c|c|}
\hline Process & References & $\tau_{\mathrm{QZE}}$ & $\tau_{\mathrm{AZE}}$ \\
\hline Radiative decay in a cavity & Kofman and Kurizki [14] & ns & ns \\
\hline Radiative decay in open space & Kofman and Kurizki [12] & does not exist & $\begin{array}{l}\text { ps (Rydberg transition) } \\
\text { fs (optical transition) }\end{array}$ \\
\hline $\begin{array}{l}\text { Photon polarization decay via random } \\
\text { modulation in a cavity }\end{array}$ & Kofman, Kurizki, and Opatrny [24] & $10 \mathrm{~ns}$ & $10 \mathrm{~ns}$ \\
\hline Transmission of tunneling emitting atoms & Japha and Kurizki [25] & $?$ & ns \\
\hline $\begin{array}{l}\text { Atomic tunneling (escape) from "washboard" } \\
\text { (accelerated) periodic potential }\end{array}$ & Wilkinson et al. [23] & $0.01 \mu \mathrm{s}$ & $\mu \mathrm{s}$ \\
\hline $\begin{array}{l}\text { Electron tunneling in current-biased SQUID } \\
\text { ("washboard" potential) }\end{array}$ & Silvestrini et al. [26] & ? & $10 \mathrm{~ns}$ \\
\hline Nuclear $\beta$-decay & Kofman and Kurizki [12] & does not exist & $10^{-18} \mathrm{~s}$ \\
\hline Near-threshold photodetachment & Lewenstein and Rza̧żewski [15] & fs & $\mathrm{ms}$ \\
\hline
\end{tabular}

resonance) reservoir, such as radiative or photonpolarization decay in a cavity.

\section{Conclusions}

Our simple universal formula (4) results in general criteria for the QZE:

(i) It can only occur in systems with spectral width below the resonance energy.

(ii) It is principally unattainable in open-space radiative or nuclear $\beta$-decay, because the required mea-

[1] L. A. Khalfin, JETP Lett. 8, 65 (1968).

[2] L. Fonda, G. C. Ghirardi, A. Rimin, and T. Weber, Nuovo Cim. 15A, 689 (1973).

[3] B. Misra and E. C. G. Sudarshan, J. Math. Phys. 18, 756 (1977).

[4] J. J. Sakurai, Modern Quantum Mechanics, AddisonWesley, Reading 1994, pp. 484-486.

[5] E. Joos, Phys. Rev. A 29, 1626 (1984).

[6] R. J. Cook, Physica Scripta T21, 49 (1988).

[7] W. M. Itano, D. J. Heinzen, J. J. Bollinger, and D. J. Wineland, Phys. Rev. A 41, 2295 (1990).

[8] P. L. Knight, Nature (London) 344, 493 (1990).

[9] V. Frerichs, and A. Schenzle, Phys. Rev. A 44, 1962 (1991).

[10] L. S. Schulman, Phys. Rev. A 57, 1509 (1998).

[11] P. Facchi and S. Pascazio, Phys. Lett. A 241, 139 (1998).

[12] A. G. Kofman and G. Kurizki, Nature (London) $\mathbf{4 0 5}$, 546 (2000).

[13] Related effects have been noted in proton decay by A. M. Lane, Phys. Lett. 99A, 359 (1983) and recently by us [14], for radiative decay in cavities, and in photodetachment in [15].

[14] A. G. Kofman and G. Kurizki, Phys. Rev. A 54, R3750 (1996). surement rates would cause the creation of new particles.

(iii) Contrary to the widespread view, frequent measurements can be chosen to accelerate essentially any decay process. Hence, the anti-Zeno effect should be far more ubiquitous than the QZE.

A variety of systems have been shown to be promising candidates for experimental studies of the AZE, which is almost always much more accessible than the QZE.

[15] M. Lewenstein and K. Rzạżewski, Phys. Rev. A 61, 022105 (2000).

[16] P. W. Milonni, Nature (London) 405, 525 (2000).

[17] C. Seife, Science 288, 1564, (2000).

[18] Handbook of Mathematical Functions, Natl. Bur. Stand. Appl. Math. Ser. No. 55, edited by M. Abramowitz and I. A. Stegun (U.S. GPO, Washington, DC, 1964).

[19] L. S. Schulman, J. Phys. A 30, L293 (1997).

[20] P. Facchi, H. Nakazato, and S. Pascazio, quant$\mathrm{ph} / 0006094$.

[21] H. E. Moses, Lett. Nuovo Cim. 4, 51 (1972).

[22] This is a modification of the setup used by P. G. Kwiat, Physica Scripta T76, 115 (1998).

[23] S. R. Wilkinson, C. S. Bharucha, M. S. Fischer, K. W. Madison, P. R. Morrow, Q. Niu, B. Sundaram, and M. G. Raizen, Nature (London) 387, 575 (1997); Q. Niu and M. G. Raizen, Phys. Rev. Lett. 80, 3491 (1998).

[24] A. G. Kofman, G. Kurizki, and T. Opatrný, Phys. Rev. A (in press).

[25] Y. Japha and G. Kurizki, Phys. Rev. Lett. 77, 2909 (1996); ibid., 80, 3739 (1998).

[26] P. Silvestrini, V. G. Palmieri, B. Ruggiero, and M. Russo, Phys. Rev. Lett. 79, 3046 (1997). 